\title{
CAPACITÉ DE LA MÉTHODE DES MICROHABITATS A PRÉDIRE L'HABITAT DE REPRODUCTION DE LA TRUITE COMMUNE.
}

\author{
M. DELACOSTE, P. BARAN, J.M. LASCAUX, G. SEGURA et A. BELAUD
}

Laboratoire d'Ingénierie Agronomique, Equipe Environnement Aquatique et Aquaculture, ENSAT, 145 av. de Muret, 31076 Toulouse Cedex, France.

\section{RÉSUMÉ}

La méthode des microhabitats a été appliquée pour le stade reproduction de la truite commune sur 205 faciès d'écoulement, appartenant à 6 rivières des Pyrénées centrales. La relation entre Surface Pondérée Utile (SPU) et densité de frayères est meilleure avec la courbe d'utilisation d'habitat développée sur les rivières pyrénéennes qu'avec celle proposée par BOVEE (1978). Cependant, la SPU est importante pour certains types de faciès présentant de faibles densités de frayères. Ceci se traduit par un effet faciès au niveau du taux d'utilisation de la SPU. Ce phénomène est dû à une surestimation de la SPU pour certains types de faciès où le gravier, dispersé entre des matériaux grossiers et donc inutilisable par la truite pour se reproduire, est pris en compte lors du calcul de la SPU. Cette étude souligne l'importance du caractère régional des courbes d'utilisation d'habitat, mais surtout la nécessité de prendre en compte un critère d'agrégation des matériaux lors de la description du substrat, dans l'optique de décrire l'habitat de reproduction des salmonidés.

Mots-clés : méthode des microhabitats, truite, reproduction.

\section{ABILITY OF INSTREAM FLOW INCREMENTAL METHODOLOGY TO PREDICT BROWN TROUT SPAWNING HABITAT.}

\section{ABSTRACT}

The Instream Flow Incremental Method was applied to brown trout reproduction, in 205 morphodynamic units of 6 Pyrenees mountain streams. The correlation between Weighted Usable Area (WUA) and redd density was higher when using a local habitat suitability curve, instead of the standard curve of BOVEE (1978). However, WUA was high in some units with low redd density. This was due to an over-estimation of the WUA in some types of unit where the gravel taken into account in WUA calculation was scattered between coarse material and thus unusable for trout spawning. This study underlines the importance of using an aggregation criterion when describing the substratum for spawning salmonids.

Key-words : Instream Flow Incremental Methodology, trout, reproduction.

\section{INTRODUCTION}

La méthode des microhabitats, développée pour résoudre des problèmes de gestion de débit (STALNAKER et ARNETTE, 1976), est de plus en plus utilisée dans le but de prédire la capacité d'accueil physique des cours d'eau à truite, à partir de la vitesse du courant, de la hauteur d'eau et du substrat.

Diverses études ont tenté de mettre en relation les prédictions de cette méthode et des caractéristiques de populations salmonicoles, certaines avec succès (STALNAKER, 1979 ; SOUCHON et al., 1989 ; JOWETT, 1992 ; BARAN et al., 1993a), d'autres non (CONDER et ANNEAR, 1987 ; SHIRVELL et MORANTZ, 1983 ; SHIRVELL, 1989 ; POUILLY et SOUCHON, 1995). 
Les principales critiques émises concernant la méthode des microhabitats sont :

1) les courbes de préférence d'habitat issues de pêches électriques sont représentatives de plusieurs activités (cache et nutrition) (SHIRVELL, 1986),

2) les courbes de préférence d'habitat présentent des variabilités spatio-temporelles (HEGGENES, 1994),

2) la dynamique de l'habitat n'est généralement pas prise en compte (LOAR et al., 1985),

3) des variables importantes vis-à-vis des populations piscicoles ne sont pas prises en compte, telles que la température (FERGUSON, 1958 in SHIRVELL, 1986), la productivité du milieu (SHIRVELL, 1986), les caches (BINNS et EISERMAN, 1979), ou la compétition (FAUSCH et WHITE, 1981),

4) l'hypothèse de non-indépendance des variables vitesse, profondeur et substrat vis-à-vis du microhabitat utilisé par un poisson n'est pas vérifiée (ORTH et MAUGHAN, 1982 ; SHIRVELL et DUNGEY, 1983).

Peu d'études ont testé cette méthode pour la phase de reproduction de la truite commune. Elle permet pourtant d'éliminer plusieurs critiques précédemment évoquées. La période de reproduction est courte, et lorsque les débits y sont stables, une image instantanée de l'habitat devrait suffir à décrire la capacité d'accueil physique du milieu. Les courbes de préférence d'habitat de reproduction sont basées sur des mesures de frayères, et représentent donc uniquement l'activité de reproduction. Enfin, le substrat, la vitesse de courant et la hauteur d'eau sont des variables majeures quant au choix du microhabitat de reproduction de la truite commune (REISER et WESCHE, 1977).

Le but de cette étude est donc d'appliquer la méthode des microhabitats pour la phase reproduction de la truite commune (Salmo trutta L.), dans le but de tester :

1) la relation existant entre la Surface Pondérée Utile et la densité de frayères,

2) l'effet d'une courbe régionale sur le calcul de la SPU, par rapport à la classique courbe de BOVEE (1978),

3) comparer la SPU à un autre descripteur de l'habitat de reproduction, la Surface de Granulométrie Favorable à la reproduction de la truite (DELACOSTE et al., 1993).

\section{MATÉRIEL ET MÉTHODE}

L'étude a été réalisée sur 6 rivières des Pyrénées centrales (la Pique, le Lys, l'One, la Neste du Louron, l'Adour de Lesponne et le Gave d'Arrens), de pente variant de 5,9 à 17,8\%, de numéro d'ordre compris entre 3 et 4 , et à régime nival. L'altitude des stations d'étude varie entre 470 et $1117 \mathrm{~m}$, et leur pente de 0,8 à $7,5 \%$. Leurs peuplements piscicoles sont constitués de truite commune (Salmo trutta L.) et de chabot (Cottus gobio L.).

\section{Mesures d'habitat}

Vingt-neuf stations réparties sur les 6 rivières ont été étudiées. Chaque station a été divisée en faciès d'écoulement (MALAVOI, 1989). Sur chacun de ces faciès, des transects de hauteur d'eau (sondage au $\mathrm{cm}$ près) et de vitesse du courant (mesurée au $\mathrm{cm} / \mathrm{s}$ près, à l'aide d'un courantomètre Marsh McBirney 201D) ont été effectués, et la granulométrie a été estimée au point contact selon l'échelle de CAILLEUX (1954). La vitesse de courant a été mesurée au $2 / 3$ de la profondeur, et également à $5 \mathrm{~cm}$ du fond. Le nombre de transects par faciès a été déterminé selon les recommandations de PARENT (1991) (en moyenne un transect tous les $5.5 \mathrm{~m}$ ). Le débit n'a pas changé entre la période de reproduction (novembre-décembre) et la date des mesures d'habitat (janvier).

Parallèlement à ces mesures, le pourcentage de caches, et la surface granulométriquement favorable à la reproduction (SGF, surface du faciès occupée par une granulométrie de 0,2 à $5 \mathrm{~cm}$ de diamètre, ramenée au mètre de berge (DELACOSTE et al., 1993)) ont été mesurés sur chaque faciès. Le comptage des frayères de truites (nids) a été effectué selon la méthodologie employée par DELACOSTE et al. (ibidem). L'abondance de frayères est exprimée en densité par unité de longueur. 


\section{Construction des courbes d'utilisation de l'habitat}

Sept cent quatre-vingt-cinq frayères de truite commune ont été mesurées sur 6 rivières. La profondeur avant creux, la vitesse avant creux à $5 \mathrm{~cm}$ du fond, et la granulométrie environnant la frayère mais non remaniée par la truite, ont été mesurées pour chaque frayère. Les caractéristiques d'habitat mesurées ne sont donc pas influencées par les structures de la frayère. La vitesse au fond a été préférée à la vitesse au $2 / 3$ de la profondeur, car elle correspond à la vitesse ressentie par la truite lors du choix de son emplacement de reproduction (SHIRVELL et DUNGEY, 1983).

Les courbes ont été établies par une analyse des fréquences, par la formule:

$$
\begin{aligned}
F i=n i / n \text { avec } F i & : \text { fréquence de la classe } i \\
n i & : \text { nombre de frayères dans la classe } i \\
n & : \text { nombre total de frayères mesurées. }
\end{aligned}
$$

Le nombre de frayères mesurées pour chaque rivière n'étant pas égal, les fréquences d'utilisation ont été calculées pour chaque rivière, puis sommées et normées à 1 , afin de donner le même poids à toutes les rivières.

\section{Traitement des données}

Le calcul de la Surface Pondérée Utile (SPU) a été effectué selon le protocole établi par BELAUD et al. (1989). Deux valeurs de SPU ont été calculées : la première à partir des courbes d'utilisation d'habitat de BOVEE (1978) et de la vitesse au 2/3 de la profondeur, la seconde à partir des courbes d'utilisation d'habitat construites dans cette étude, et de la vitesse au fond. La SPU a été exprimée en $\mathrm{m}^{2}$ par $m$ de berge.

La normalité des données a été vérifiée par le test de LILLIEFORS (SPRENT, 1992). Dans le cas de données non normales, la liaison entre 2 variables a été quantifiée par le coefficient de corrélation de rang Rs de SPEARMAN, et les différences de caractéristiques des groupes de faciès ont été mises en évidence grâce au test de KRUSKALL-WALLIS (SPRENT, 1992).

\section{RÉSULTATS}

\section{Courbe d'utilisation d'habitat}

Les courbes d'utilisation d'habitat construites dans les rivières Pyrénéennes diffèrent de celles données par BOVEE (1978) (Figure 1). Les courbes de hauteur d'eau sont semblables jusqu'à $30 \mathrm{~cm}$. Au-delà de cette valeur, la courbe des Pyrénées chute brutalement, alors que la courbe de BOVEE marque un plateau entre 45 et $70 \mathrm{~cm}$. La hauteur d'eau maximale utilisée est de $50 \mathrm{~cm}$ pour les Pyrénées, et $85 \mathrm{~cm}$ pour BOVEE.

Les courbes de vitesse de courant sont très différentes. Les vitesses de la courbe des Pyrénées sont plus faibles que celles de la courbe de BOVEE. Les classes de vitesse les plus utilisées sont $25-30 \mathrm{~cm} / \mathrm{s}$ dans les Pyrénées, contre $45-60 \mathrm{~cm} / \mathrm{s}$ pour la courbe de BOVEE.

Le substrat utilisé est le même pour les 2 courbes : graviers et petits galets.

\section{Relation SPU - densité de frayères}

Les valeurs de SPU $\left(\mathrm{m}^{2} / \mathrm{m}\right)$ diffèrent significativement selon le type de faciès considéré (KRUSKALL-WALLIS, $p<0,01$ ), de même que la densité de frayères (KRUSKALL-WALLIS, $p<0,01$ ) (Tableau I).

II existe une relation significative entre la SPU calculée et la densité de frayères (Figure 2). La corrélation est cependant plus forte avec la courbe développée dans les Pyrénées (Rs $=0,51 ; p<0.001)$ qu'avec la courbe de BOVEE (Rs $=0,39 ; p<0.001$ ). Par conséquent, nous n'utiliserons plus, dorénavant, que la SPU calculée à partir de la courbe des Pyrénées.

La relation SPU - densité de frayères n'est pas la même selon le type de faciès considéré (Figure 3). L'augmentation de densité de frayères en réponse à une augmentation de SPU est beaucoup plus lente pour certains groupes (rapides, escaliers, radiers), que pour d'autres (plats, mouilles). 

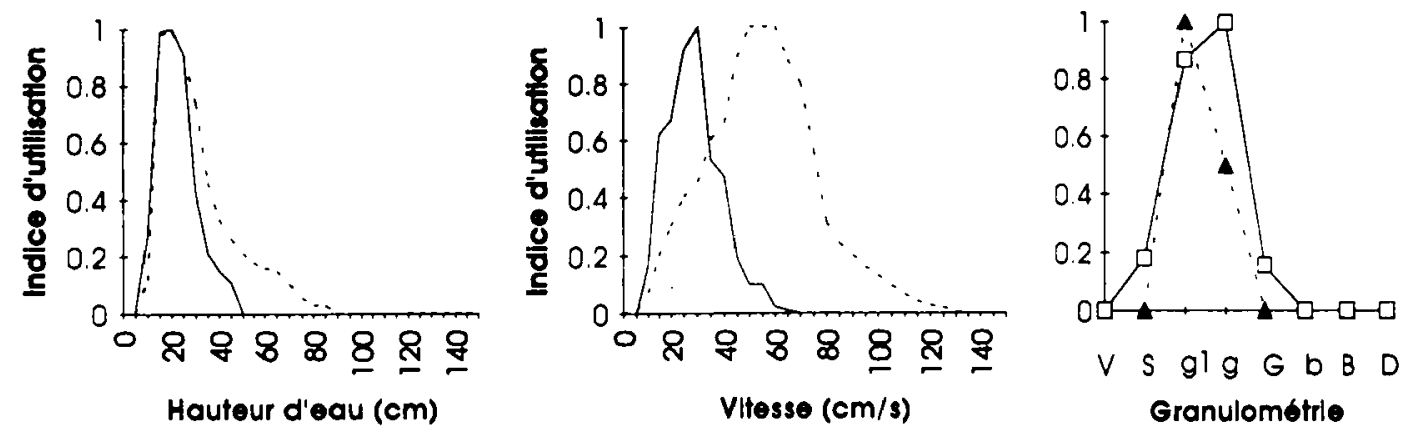

Figure 1 : Courbes d'utilisation d'habitat pour le stade reproduction, développées dans cette étude (trait continu ou carrés) et par BOVEE (1978) (pointillé ou triangles). La vitesse est mesurée aux $2 / 3$ de la profondeur pour BOVEE et à $5 \mathrm{~cm}$ du fond pour cette étude. Le substrat est défini en $\mathrm{V}$ : vase $(<0.005 \mathrm{~cm}), \mathrm{S}$ : sable $(0.005-0.2 \mathrm{~cm}), \mathrm{g} 1$ : graviers $(0.2-2 \mathrm{~cm}), \mathrm{g}$ : petits galets $(2-10 \mathrm{~cm}), \mathrm{G}$ : gros galets $(10-20 \mathrm{~cm})$, b : petits blocs $(20-60 \mathrm{~cm})$, $B$ : gros blocs $(>60 \mathrm{~cm}), D:$ dalle $(>60 \mathrm{~cm}$ et non protubérant).

Figure 1 : Habitat suitability curves for reproduction, developed in the Pyrenees Mountains (solid line and square) and by BOVEE (1978) (broken line and triangle). Substrat is $V$ : silt $(<0.005 \mathrm{~cm}), S$ : sand $(0.005-0.2 \mathrm{~cm})$, g1 : gravel $(0.2-2 \mathrm{~cm}), \mathrm{g}$ : small cobble $(2-10 \mathrm{~cm}), \mathrm{G}$ : big cobbles $(10-20 \mathrm{~cm})$, b : small boulders $(20-60 \mathrm{~cm}), B$ : large boulders $(>60 \mathrm{~cm}), D$ : flag stones $(>60 \mathrm{~cm})$.

Tableau I : Surface Pondérée Utile (SPU), et taux d'occupation de la SPU (nb de frayères $/ \mathrm{m}^{2}$ de SPU), (moyenne et écart-type), calculés à partir des courbes de BOVEE (1978) et de celles développées dans cette étude, et densité moyenne de frayères par mètre.

Table I : Weighted Usable Area and number of redds/WUA (mean and standard deviation), using BOVEE (1978) suitability curves and the curves developed in the Pyrenees Mountains, and redd density per metre.

\begin{tabular}{lccccc}
\hline $\begin{array}{l}\text { Type de } \\
\text { faciès }\end{array}$ & \multicolumn{2}{c}{ SPU $\left(\mathrm{m}^{2} / \mathrm{m}\right)$} & \multicolumn{2}{c}{ Toux d'Occupation de la SPU } & Densité de \\
\hline Mouille & $0.16(0.16)$ & $0.26(0.32)$ & $5.90(6.40)$ & $3.15(2.93)$ & $0.70(0.71)$ \\
Plat & $0.30(0.48)$ & $0.38(0.40)$ & $5.39(10.20)$ & $2.64(3.65)$ & $0.75(0.86)$ \\
Radier & $0.35(0.50)$ & $0.53(0.60)$ & $5.44(22.71)$ & $0.96(1.18)$ & $0.52(0.67)$ \\
Rapide & $0.49(0.48)$ & $0.39(0.34)$ & $0.34(0.62)$ & $0.30(0.49)$ & $0.12(0.19)$ \\
Cascade & $0.08(0.09)$ & $0.13(0.12)$ & $0.55(1.23)$ & $0.45(1.01)$ & $0.04(0.07)$ \\
\hline
\end{tabular}



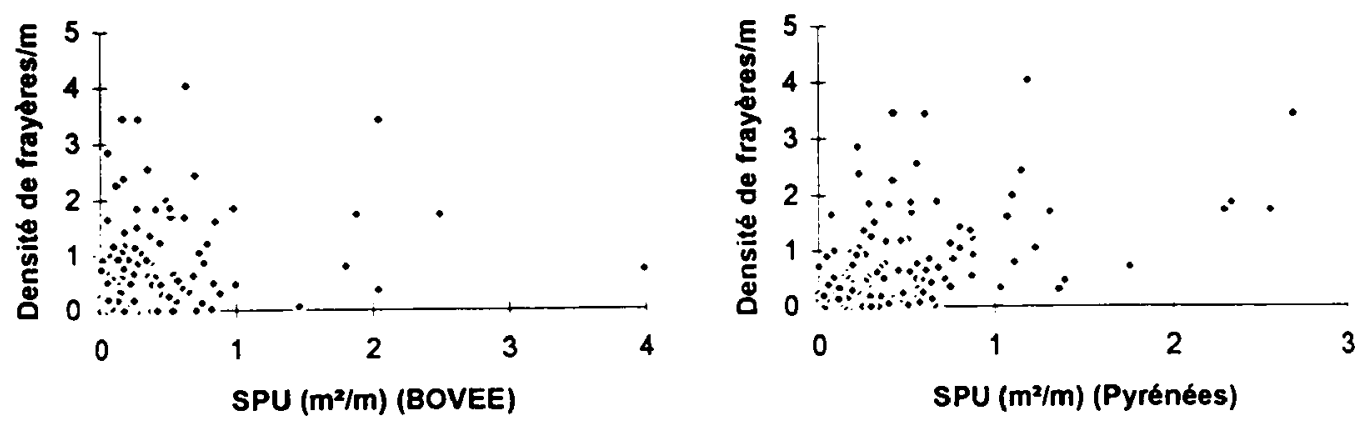

Figure 2 : Relation SPU $\left(\mathrm{m}^{2} / \mathrm{m}\right)$ et densité de frayères (courbe de BOVEE et courbe Pyrénées).

Figure 2 : Relationship between WUA $\left(\mathrm{m}^{2} / \mathrm{m}\right)$ and redd density (BOVEE curve and Pyrenees curve).

MOUILLES

RAPIDES
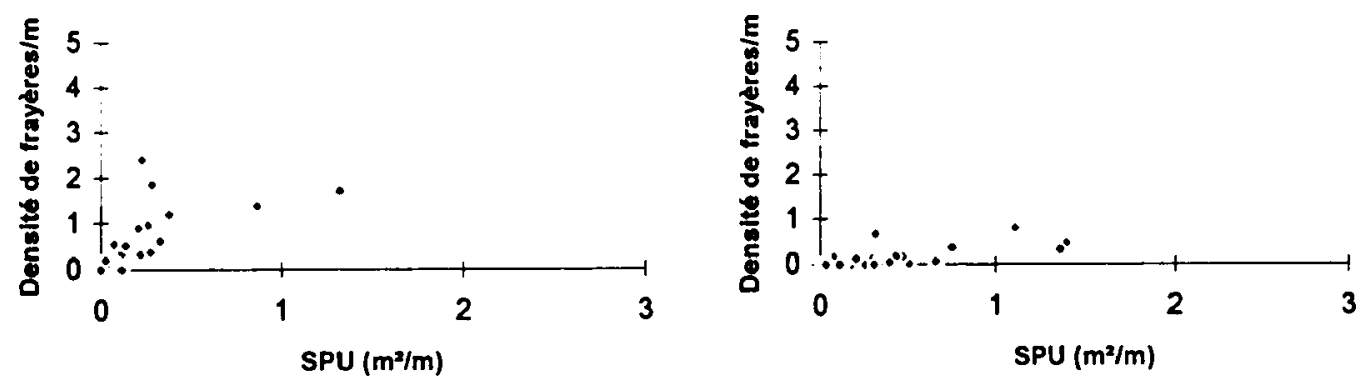

PLATS

RADIERS
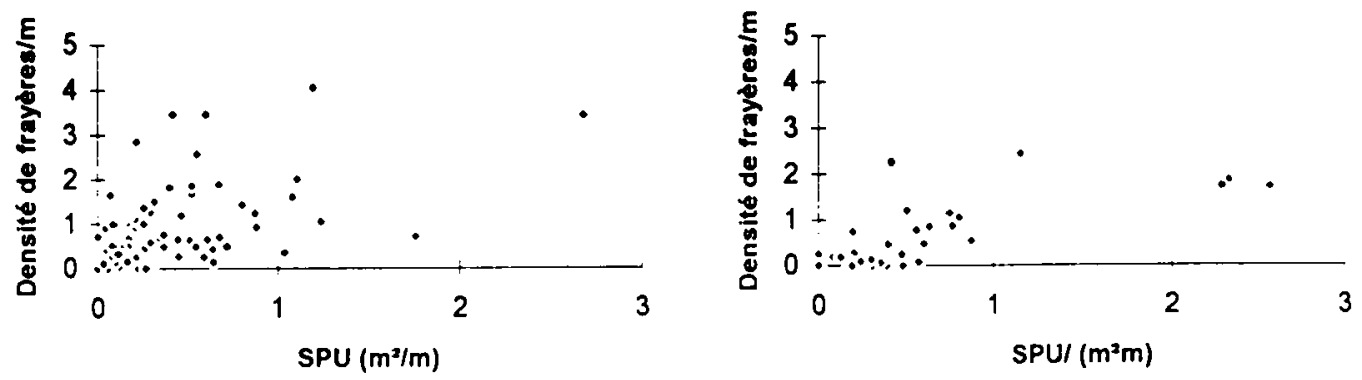

Figure 3 : Relation SPU $\left(\mathrm{m}^{2} / \mathrm{m}\right)$ et densité de frayères pour les différents types de faciès.

Figure 3 : Relationship between Weighted Usable Area $\left(\mathrm{m}^{2} / \mathrm{m}\right)$ and redd density for each type of morphodynamic unit. 
Les taux d'occupation moyens de l'habitat (nombre de frayères/SPU) sont significativement différents selon le type de faciès considéré (KRUSKALL-WALLIS, $p<0,01$ ) (Tableau I). Ils ne diffèrent pas, par contre, entre les différentes stations ou les différentes rivières (KRUSKALL-WALLIS).

II n'existe pas de relation significative entre le taux d'occupation et le pourcentage de caches ou la largeur du faciès.

La Surface de Granulométrie Favorable à la reproduction de la truite est très significativement corrélée à la densité de frayères ( $R s=0.69 ; p<0.001$ ). Elle représente la surface maximale de substrat utilisable par la truite pour se reproduire. Elle est inférieure à la SPU pour la majorité des rapides, des radiers, des cascades, et pour certains plats. Elle est par contre supérieure à la SPU pour les faciès profonds (mouilles, plats profonds) et certains types de plats.

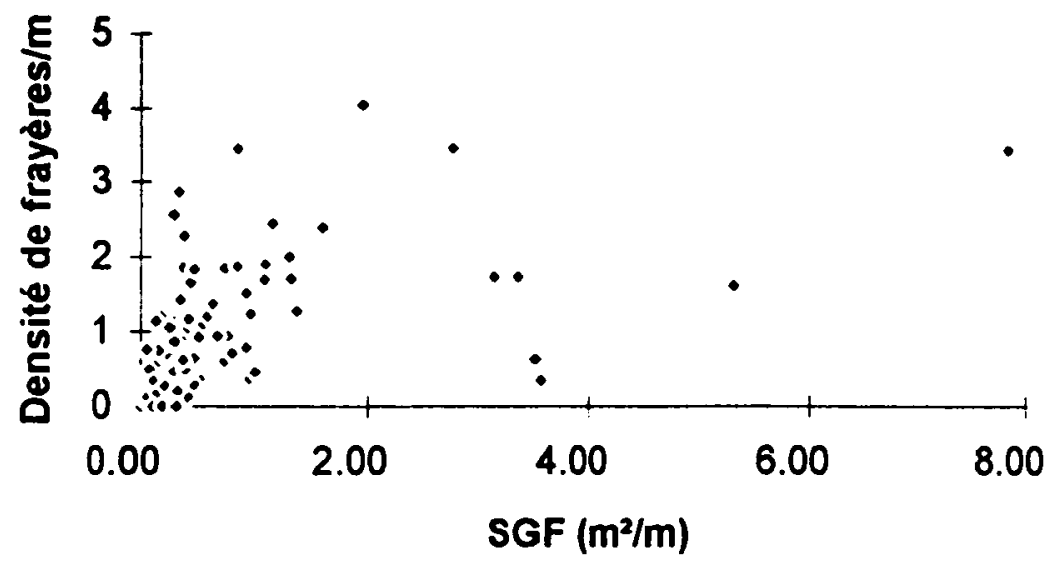

Figure 4 : Relation Surface de Granulométrie Favorable à la reproduction de la truite $\left(\mathrm{m}^{2} / \mathrm{m}\right)$ et densité de frayères par mètre.

Figure 4 : Relationship between surface of gravel suitable for trout spawning $\left(\mathrm{m}^{2} / \mathrm{m}\right)$ and redd density per metre.

\section{DISCUSSION}

\section{Courbe d'utilisation d'habitat}

Trois hypothèses peuvent expliquer les différences que l'on peut observer entre les courbes d'utilisation d'habitat construites dans les Pyrénées et celles de BOVEE :

- l'hydromorphodynamisme des rivières : les rivières pyrénéennes dans lesquelles ont été construites les courbes sont de petite taille (numéro d'ordre de 3 à 4) et très pentues. Les hauteurs d'eau importantes y sont peu fréquentes. Les dépôts de graviers sont peu abondants, et souvent associés à des petits galets. Les courbes données par BOVEE couvrent une gamme plus importante de types de cours d'eau, et donc de gamme d'habitat disponible.

- l'emplacement des mesures de la vitesse de courant : la vitesse aux $2 / 3$ de la profondeur est généralement supérieure à la vitesse au fond, et ce d'autant plus que la hauteur d'eau augmente.

- la taille des géniteurs : elle est relativement faible dans les cours d'eau étudiés, et peut constituer une hypothèse valable quant aux différences observées. Les plus gros géniteurs ont en effet tendance à utiliser des vitesses plus importantes (OTTAWAY et al., 1981). 


\section{Relation SPU - densité de frayères}

La courbe d'utilisation d'habitat établie dans les Pyrénées, et basée sur des mesures de vitesse au fond, permet une meilleure prédiction de l'habitat disponible dans les rivières étudiées. L'application d'une courbe à caractère régional permet une amélioration significative de la relation SPU-densité de frayères.

On constate cependant un effet faciès au niveau du taux d'utilisation de l'habitat. Deux hypothèses peuvent être avancées pour l'expliquer. La première est d'ordre écologique. On pourrait penser que les conditions d'habitat autres que celles de la reproduction, offertes aux géniteurs par le faciès, jouent un rôle sur son utilisation lors de la reproduction. En effet, les faciès les plus occupés sont les mouilles et les plats, faciès favorables aux adultes (BARAN et al., 1993b). Cependant, il n'existe pas de relation significative entre le taux d'occupation de la SPU d'un faciès, et la SPU pour le stade adulte, ou le pourcentage de caches de ce faciès (caches totales ou sous-caves). La seconde hypothèse est d'ordre méthodologique. La granulométrie est, en effet, un facteur clé quant au choix du microhabitat de reproduction par la truite (SHIRVELL et DUNGEY, 1983 ; WITZEL et Mac CRIMMON, 1983). Ce facteur est parfois difficile à estimer. Or, une faible variation de la granulométrie peut entraîner des variations importantes de résultats. Dans le type de cours d'eau étudié, les zones favorables sont souvent dispersées au sein du faciès, et de petite taille. Le problème de la représentativité granulométrique du transect se pose alors si l'on ne veut pas multiplier leur nombre à l'infini. De plus, définir la granulométrie uniquement en pourcentage de classe de taille n'est pas suffisant lorsqu'on s'intéresse à la reproduction. En effet, un bon pourcentage de graviers disséminés entre des galets ne sera pas utilisé par la truite, alors que ce même pourcentage agrégé en une surface homogène aura de fortes probabilités d'être utilisé. Ces 2 critères (classe de taille et surface de granulométrie homogène) sont pris en compte par la variable Surface de Granulométrie Favorable. La SGF, quantifiant toutes les zones de substrat favorable, représente la surface maximale utilisable. Si la SPU est supérieure à la SGF, cela nous indique qu'elle est surestimée. C'est le cas des rapides, des radiers, des cascades et d'une partie des plats. Un pourcentage non négligeable de graviers dispersés dans des matériaux plus grossiers, et donc inutilisables par la truite, mais pris en compte lors du calcul de la SPU du fait du mode de description du substrat, est responsable de cette surestimation. Si, par contre, la SPU est plus faible que la SGF, cela nous indique qu'une partie de la SGF est inutilisée. C'est le cas des faciès profonds (mouilles, plats profonds) et d'une partie des plats, possédant souvent des dépôts de graviers dans des zones de vitesse nulle ou de très faible profondeur, et donc peu favorables.

L'effet faciès mis en évidence est donc principalement dû à une surestimation de la SPU du fait d'une méthodologie de description de la granulométrie inadaptée à la phase reproduction de la truite. Un couplage de la méthode des microhabitats et de la SGF (en réalisant les transects sur la SGF) pourrait représenter une bonne méthodologie de description de l'habitat de reproduction des salmonidés.

\section{CONCLUSION}

Cette étude met l'accent sur deux points importants concernant la méthode des microhabitats

- la particularité régionale des courbes d'utilisation d'habitat, qui peut améliorer les relations SPU - variable biologique de façon significative,

- la nécessité de tenir compte du degré d'agrégation des matériaux lors de la description de l'habitat de reproduction de la truite, et des salmonidés en général.

\section{BIBLIOGRAPHIE}

BARAN P., DELACOSTE M., LASCAUX J.M., BELAUD A., 1993a. Relations entre les caractéristiques de l'habitat et les populations de truites communes (Salmo trutta L.) de la vallée de la Neste d'Aure. Bull. Fr. Pêche Piscic., 331, 321-340. 
BARAN P., DAUBA F., DELACOSTE M., LASCAUX J.M., 1993b. Essais d'évaluation quantitative du potentiel halieutique d'une rivière à Salmonidés à partir des données de l'habitat physique. In GASCUEL D., DURAND D., FONTENEAU G., Les recherches françaises en évaluation quantitative et modélisation des ressources et systèmes halieutiques, 15-38, éditions de l'ORSTOM, Colloque et Séminaire, Paris.

BELAUD A., CHAVEROCHE P., LIM P., SABATON C., 1989. Probability-of-use curves applied to brown trout (Salmo trutta fario L.) in rivers of southern France. Regulated Rivers : research and management, 3, 321-336.

BINNS N.A., EISERMAN F.M., 1979. Quantification of fluvial trout habitat in Wyoming. Trans. Am. Fish. Soc., 108 (3), 215-228.

BOVEE K.D., 1978. Probability-of-use criteria for the family salmonidae. Report U.S. Fish and Wildlife Service, FWS/OBS-78/07, Fort Collins, Colorado, U.S.A., 80 p.

CAILLEUX A., 1954. Limites dimensionnelles des noms des fractions granulométriques. Bull. Soc. Géol. Fr., 4, 643-646.

CONDER A.L., ANNEAR T.C., 1987. Test of weighted usable area derived from a PHABSIM model for instream flow studies on trout streams. N. Am. J. Fish. Manage., 7, 339-350.

DELACOSTE M., BARAN P., DAUBA F., BELAUD A., 1993. Etude du macrohabitat de reproduction de la truite commune (Salmo trutta $L$.) dans une rivière pyrénéenne, la Neste du Louron. Evaluation d'un potentiel de l'habitat physique de reproduction. Bull. Fr. Pêche Piscic., 331, 341-356.

FAUSCH K.D., WHITE R.J., 1981. Competition between brook trout (Salvelinus fontinalis) and brown trout (Salmo trutta) in a Michigan stream. Can. J. Fish Aquat. Sci., 38, 1220-1227.

HEGGENES J., 1994. Physical habitat selection by brown trout (Salmo trutta) and young Atlantic salmon (S. salar) in spatially and temporally heterogeneous streams : implications for hydraulic modelling. Proceedings of the 1st international Symposium on Habitat Hydraulics, Trondheim, Norvège, 12-30.

JOWETT I.G., 1992. Models of the abundance of large brown trout in New Zealand Rivers. North Am. J. Fish. Manage., 12, 417-432.

LOAR J.M., SALE M.J., CADA G.F., 1985. Application of habitat evaluation in southern Appalachian trout stream. Environmental Sciences Division publication, 2383, ORNLTM. 9323, 230 p.

MALAVOI J.R., 1989. Typologie des faciès d'écoulement ou unités morpho dynamiques d'un cours d'eau à haute énergie. Bull. Fr. Pêche Piscic., 315, 189-210.

ORTH D.J., MAUGHAN O.E., 1982. Evaluation of the incremental methodology for recommending instream flow for fishes. Trans. Am. Fish. Soc., 111, 413-445.

OTTAWAY E.M., CARLING P.A., CLARKE A., READER N.A., 1981. Observations on the structure of brown trout, Salmo trutta Linnaeus, redds. J. Fish Biol., 19, 593-607.

PARENT A., 1991. Etude de protocoles expérimentaux d'analyses de la qualité d'habitat piscicole d'un tronçon de rivière à débit réservé. Rapport D.E.R. EDF HE 31/91-05, 54 p.

POUILLY M., SOUCHON Y., 1995. Méthode des microhabitats : validation et perspectives. Bull. Fr. Pêche Piscic., 337/338/339, N Spécial "Relation entre le poisson et son habitat".

REISER D.W., WESCHE T.A., 1977. Determination of physical and hydraulic preferences of brown and brook trout in the selection of spawning locations. United States Department of the Interior, Water Resources Research Institute, Water Research and Technology Completion Report C.7002, University of Wyoming, Laramie, U.S.A, 100 p. 
SHIRVELL C.S., 1986. Pitfalls of physical habitat simulation in the instream flow incremental methodology. Can. Tech. Rep. Fish. Aquat. Sci., 1460, 68 p.

SHIRVELL C.S., 1989. Ability of PHABSIM to predict chinook salmon spawning habitat. Regulated rivers : research and management, vol. 3, 277-289.

SHIRVELL C.S., DUNGEY R.G., 1983. Microhabitats chosen by brown trout for feeding and spawning in rivers. Trans. Am. Fish. Soc., 112 (3), 355-357.

SHIRVELL C.S., MORANTZ D.L., 1983. Assessments of instream flow incremental methodology for Atlantic salmon in Nova Scotia. Trans. Can. Elect. Assoc., vol 22, 83-H-108, $22 \mathrm{p}$.

SOUCHON Y., TROCHERIE F., FRAGNOUD E., LACOMBE C., 1989. Les modèles numériques des microhabitats des poissons : applications et nouveaux développements. Rev. Scie. Eau., 2, 807-830.

SPRENT P., 1992. Pratique des statistiques non-paramétriques. INRA éditions, Paris, $294 \mathrm{p}$.

STALNAKER C., 1979. The use of habitat structure preferenda for establishing flow regimes necessary for maintenance of fish habitat. In WARD \& STANDFORD, The ecology of regulated streams, 321-337, New York, Plenum, U.S.A.

STALNAKER C.B., ARNETTE J.L., 1976. Methodologies for determination of stream resource flow requirements : an assessment. U.S. Fish and Wildlife Service FWS/OBS-76/03. Washington, D.C., U.S.A.

WITZEL L.D., Mac CRIMMON H.R., 1983. Redd site selection by brook trout and brown trout in south eastern Ontario streams. Trans. Am. Fish. Soc., 112, 760-771. 